\title{
Application of One-Dimensional Analytical Models for the Interpretation of Observations of Superconducting Fluxons
}

\author{
Giulio Pozzi $\left({ }^{1}\right)$, John E. Bonevich $\left({ }^{2,3}\right)$, Ken Harada $\left({ }^{2}\right)$, Hiroto Kasai $\left({ }^{2}\right)$, \\ Tsuyoshi Matsuda $\left({ }^{2}\right)$, Takaho Yoshida $\left({ }^{2}\right)$ and Akira Tonomura $\left({ }^{2}\right)$ \\ ( $\left.{ }^{1}\right)$ Department of Physics and Istituto Nazionale di Fisica della Materia, University of Bologna, viale \\ B. Pichat 6, 40127 Bologna, Italy \\ ( $\left.{ }^{2}\right)$ Advanced Research Laboratory, Hitachi, Ltd., Hatoyama, Saitama 350-03, Japan \\ $\left({ }^{3}\right)$ Lawrence Berkeley Laboratory, Materials Science Division, MS72-150, 1 Cyclotron Road, \\ Berkeley, CA 94720 U.S.A.
}

(Received March 1; accepted June 2, 1995)

\begin{abstract}
In order to extract quantitative information from the two-dimensional images of superconducting fluxons observed in thin tilted specimens by means of holographic or out-of-focus methods, one-dimensional line scans are taken and compared with the theoretical predictions. In particular, the trend of the reconstructed phase across the fluxon core, or the intensity distribution of its out-of-focus image have a strong similarity with those calculated by means of previous one-dimensional models, where the fluxon was considered lying perpendicular to the electron beam. This work exploits this analogy showing that, in spite of the different geometry, suitably modified one-dimensional models can be usefully applied for the interpretation of the experimental results and the analysis of the experimental conditions as well as for the assessment of new methods, like f.i. the one proposed for discriminating between London and Clem models.
\end{abstract}

\section{Introduction}

The recent success in imaging the magnetic flux-line lattice (FLL) in superconducting materials directly via transmission electron microscopy (TEM) techniques has provided a new perspective of their behaviour [1]. Information about fluxons (or vortices), contained within the electron phase, is revealed in a defocused electron micrograph whereby the fluxon appears as a spot of bright and dark contrast $[1,2]$. The dynamic behaviour of fluxons can then be observed by this Lorentz microscopy method, and their response to changes in temperature and magnetic field as well as their interaction with specimen defects like grain boundaries and dislocations can be discerned [3].

One disadvantage of Lorentz microscopy, however, is that quantitative extraction of data is problematic [4]. For instance, while Lorentz microscopy reveals the location and polarity of the 
vortex, its degree of flux quantization can not be measured. In contrast, off-axis electron holography [5] is highly quantitative since the interferometric recording of the image wavefunction by means of an electron biprism allows in the reconstruction step the recovering of both the amplitude and phase of the entire object. Therefore, it is possible both to measure the total flux enclosed by a single vortex as well as to obtain a two-dimensional distribution of the phase with high spatial resolution. These holographic data can be presented in the form of i) line scans emphasizing the trend of the phase across the fluxon or ii) two dimensional $n$-times amplified contour maps, which give an impressive view of the phase distribution and whose contour lines can be interpreted as the magnetic field lines $[6,7]$.

Whereas a former theoretical analysis (reviewing also the work done until 1972) carried out for the case of fluxons in thin specimen lying perpendicular to the electron beam predicted the feasibility of such experiments [8], the recent breakthrough is due to the fact that a new geometry has been introduced, namely the specimen is observed tilted with respect to both the vertically incident electron beam and the horizontal ancillary magnetic field used to introduce and stabilize the vortices. We note that with this geometry applied fields of up to $200 \mathrm{G}$ result in vortex spacings of about $0.5 \mu \mathrm{m}$ to $1.5 \mu \mathrm{m}$ such that each vortex may be considered as a single one.

From the wave optical point of view this case is complicated because the electrons experience both the internal and external field and the resulting phase shift is two-dimensional. Nonetheless when the fluxon is modeled by a flux tube of negligible radius, a simple analytical solution exists for the field and for the phase shift suffered by the electron beam [9]. This elementary solution can then be used as a building block to investigate more realistic models; e.g. taking into account the vortex core structure by approximating it with a suitable arrangement of flux tubes.

It has been thus shown that the most relevant features of the experimental results obtained by Lorentz microscopy $[1,2]$ and by electron holography $[6,7]$ can be interpreted by describing the magnetic field around the vortex core by means of the London or Clem models $[10,11]$. In order to appreciate the subtle differences between the predictions of these two models, it has been found that it is better to display, in addition to the two-dimensional images, also line scans taken across the fluxon core.

It turns out that these line scans have a striking similarity with those formerly calculated by means of one-dimensional models, where the fluxon was supposed to be lying in the specimen perpendicular to the electron beam. The aim of this work is to exploit this analogy, because calculations done using one-dimensional models, involving less computational and programming effort, can serve as a guide to a better understanding of the phenomena as well as to the more elaborate and time consuming two-dimensional simulations.

\section{Field Model and Associated Phase Shift}

Let us consider a single fluxon in a thin diamagnetic specimen of thickness $t$, tilted at an angle $\alpha$ with respect to the electron beam, Figure 1 . The fluxon is modelled by a bundle of flux tubes, which run perpendicular to the specimen surfaces and whose distribution, having a cylindrical symmetry around the fluxon axis $a a$, is described by one of the models developed for the bulk case.

Two models are considered in this work, the first is the London one, in which the field is described by the following equation [12],

$$
B=\frac{\Phi}{2 \pi \lambda_{\mathrm{L}}^{2}} K_{0}\left(r / \lambda_{\mathrm{L}}\right)
$$




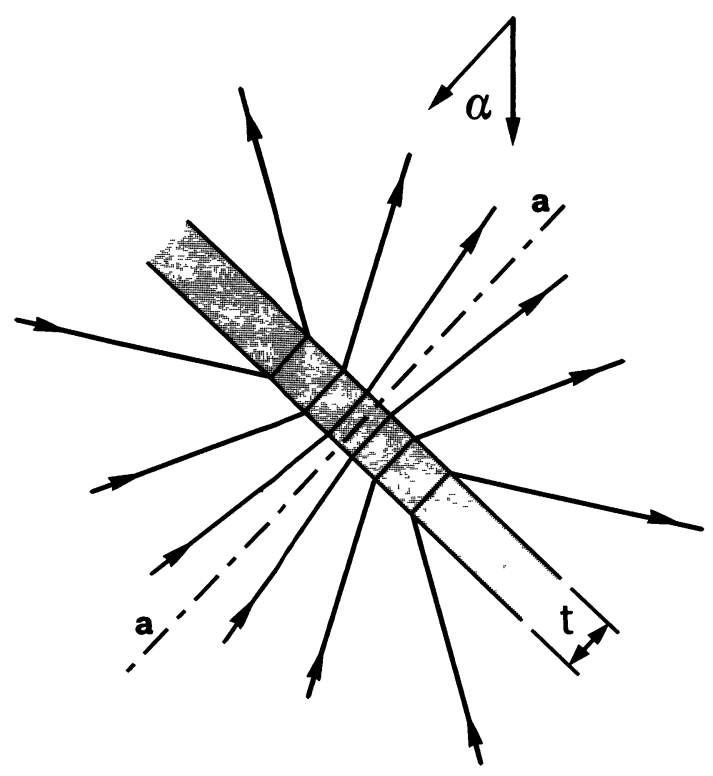

Fig. 1. - Schematic diagram for a single fluxon. The fluxon core is aligned along $a a$ within the specimen of thickness $t$ tilted by angle $\alpha$ to the electron optic axis.

where $K_{0}$ is the zero-order Bessel function,-and $r$ the radial distance from the vortex axis. The second is the Clem model, whose magnetic field distribution is given by [12]

$$
B=\frac{\Phi}{2 \pi \lambda_{\mathrm{L}} \xi_{\mathrm{v}}} \frac{K_{0}\left(R / \lambda_{\mathrm{L}}\right)}{K_{1}\left(\xi_{\mathrm{v}} / \lambda_{\mathrm{L}}\right)}
$$

where $R^{2}=r^{2}+\xi_{\mathrm{v}}^{2}$, and $K_{1}$ is the first-order Bessel function.

It should be noted that the London topography, which assumes an infinitely small normal core, is a function of a single parameter, the London penetration depth $\lambda_{\mathrm{L}}$, whereas the Clem one removes the unphysical limitation of the infinitely small normal core and is also described by an analytical expression depending on two parameters: the London penetration depth $\lambda_{\mathrm{L}}$ and the core radius $\xi_{\mathrm{v}}$, which is of the same order of magnitude as the coherence length $\xi$. Moreover, the Clem model converges to the London one when $\xi_{\mathrm{v}} \rightarrow 0$.

As an analytical expression is available for the phase shift due to a single flux tube [9], the object phase shift corresponding to a more realistic core distribution can be calculated numerically by convolving the phase shift due to the flux tube with the two-dimensional projections in the object plane of the London or Clem models. FFT methods, on a square region centered at the vortex having side $1.2 \mu \mathrm{m}$, with $256 \times 256$ sampling points, have been employed for evaluating the convolution. Although other methods of calculating the two-dimensional phase shift $\varphi(x, y)$ can be imagined, we have found our approach the easiest one for taking into account the complications arising from the tilted geometry, so essential to the success of the experimental observations

The phase shift can further be inserted into the Kirchhoff-Fresnel integral in order to calculate out-of-focus images. Here again FFT methods have been employed to calculate the image wave function in the defocus plane. In this case it should be noted that a linear phase term has been subtracted from the phase shift in order to diminish image artifacts due to Fresnel diffraction 

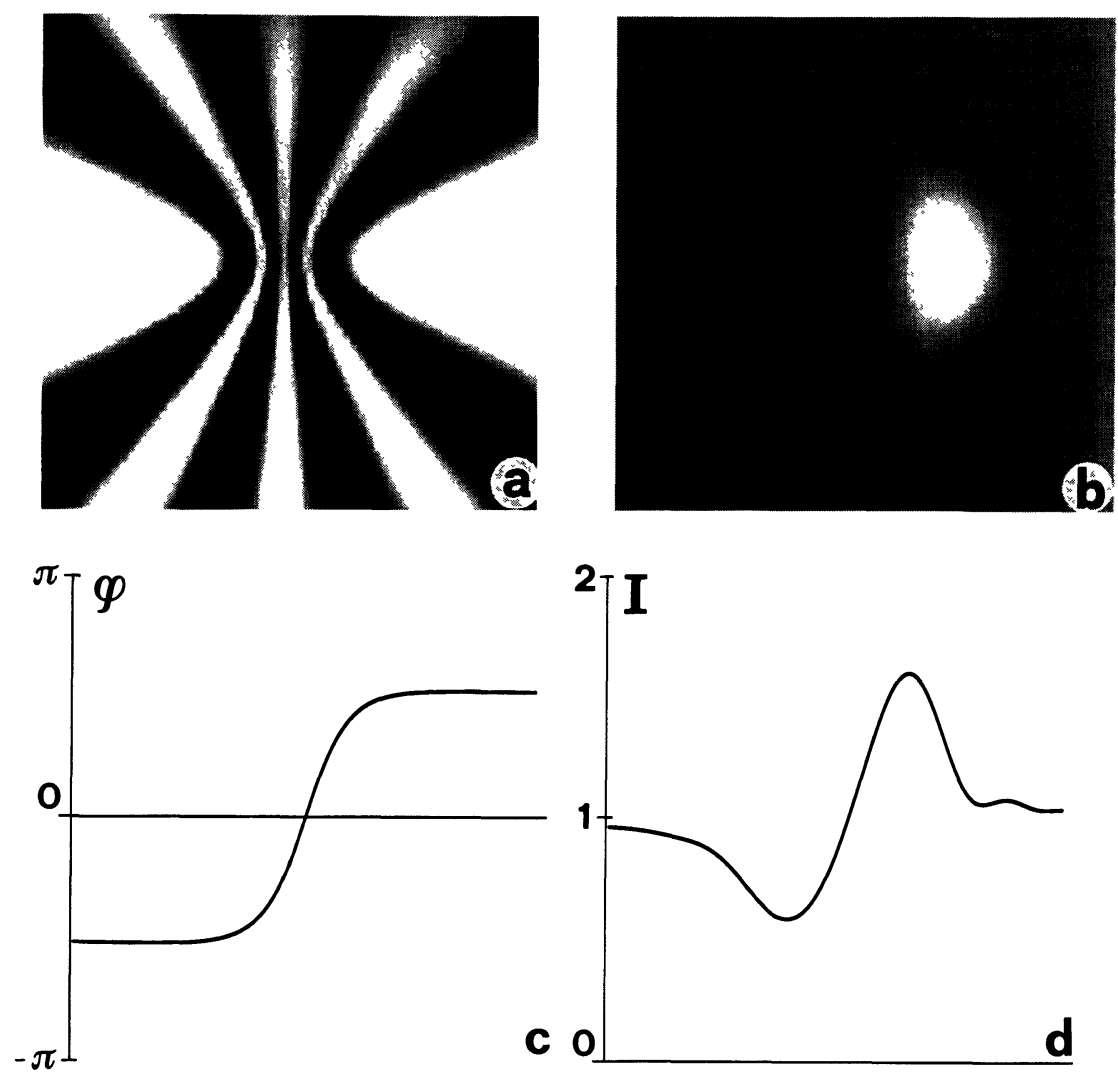

Fig. 2. - Simulation results of single vortex. The $16 \times$ phase amplified contour map (a) and its $10 \mathrm{~mm}$ defocussed image (b). Corresponding line scans across the vortex center reveal a total phase difference of $\pi / 2$ (c) and its image intensity (d). The vortex simulation parameters were $\lambda_{\mathrm{L}}=30 \mathrm{~nm}, \xi_{\mathrm{v}}=40 \mathrm{~nm}, t=30$ $\mathrm{nm}, \alpha=\pi / 4$ and the total width of the image was $20 \lambda_{\mathrm{L}}=0.6 \mu \mathrm{m}$.

effects from the edge parallel to the fluxon axis, where the phase has a jump of the order of $\pi / 2$ owing to the truncation.

Figure 2 reports typical two-dimensional results calculated for the case of a specimen having London length $\lambda_{\mathrm{L}}=30 \mathrm{~nm}$, a core radius $\xi_{\mathrm{v}}=40 \mathrm{~nm}$ (i.e. of the same order as those of the bulk Nb), thickness $30 \mathrm{~nm}$, i.e. $\lambda_{\mathrm{L}}$, tilted at an angle of $\alpha=\pi / 4$ and observed at a defocus of $10 \mathrm{~mm}$. The accelerating voltage has been taken equal to $300 \mathrm{kV}$, corresponding to a de Broglie wavelength of $\lambda_{e}=1.968 \mathrm{pm}$. Figure $2 \mathrm{a}$ reports the fluxon phase shift displayed as a 16 times amplified contour map according to the formula $I=1+\cos [16 \varphi(x, y)]$ and (b) the out-of-focus image. Below the corresponding line scans taken across the fluxon core in the symmetry plane of the unamplified phase (c) and out-of-focus image (d) are shown.

When $\alpha$ equals $\pi / 2$, that is the specimen surfaces are parallel to the electron beam, the phase shift due to the internal field can be separated from the external leakage field. In this limiting case, considering only magnetic field effects on the electron wavefunction (the specimen has a virtually infinite thickness) it turns out that the object phase shift can be calculated analytically (as shown 

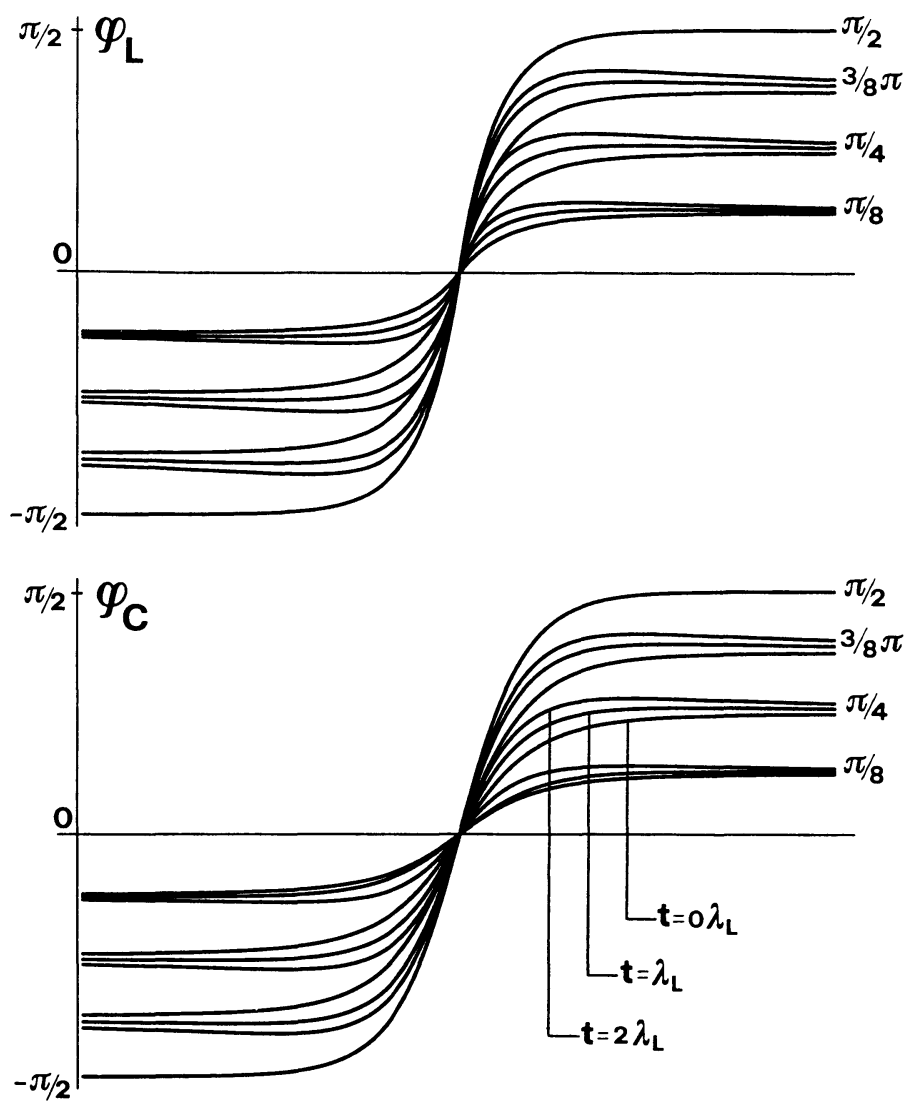

Fig. 3. - The London and Clem vortex models. Line scans of the phase across the vortex core at various thickness $\left(0 \lambda_{\mathrm{L}}, \lambda_{\mathrm{L}}, 2 \lambda_{\mathrm{L}}\right)$ and tilt angles $(\pi / 8, \pi / 4,3 \pi / 8$ and $\pi / 2)$. Larger tilts result in larger phase differences as does increasing thickness. Note that the phase for the thickest specimens contains a broad maxima. The line scan width corresponds to $20 \lambda_{\mathrm{L}}$.

by Guigay and Bourret [13]) and is given by

$$
\varphi_{\mathrm{L}}(x)=\operatorname{sign}(x) \frac{\pi}{2}\left[1-\exp \left(-\frac{|x|}{\lambda_{\mathrm{L}}}\right)\right]
$$

where $x$ is the coordinate in the object plane perpendicular to the fluxon axis.

The phase derivative, proportional to the geometric optical deflection, is given by

$$
\frac{\mathrm{d} \varphi_{\mathrm{L}}}{\mathrm{d} x}=\frac{\pi}{2 \lambda_{\mathrm{L}}} \exp \left(-\frac{|x|}{\lambda_{\mathrm{L}}}\right)
$$

For the Clem model, following the reasoning of Guigay and Bourret [13], it turns out that an analytical expression is available only for the phase derivative

$$
\frac{\mathrm{d} \varphi_{\mathrm{C}}}{\mathrm{d} x}=\frac{\pi}{2 \xi_{\mathrm{v}} K_{1}\left(\xi_{\mathrm{v}} / \lambda_{\mathrm{L}}\right)} \exp \left(-\frac{\sqrt{x^{2}+\xi_{\mathrm{v}}^{2}}}{\lambda_{\mathrm{L}}}\right)
$$

from which the phase shift can be obtained by numerical integration. 
Figure 3 reports one-dimensional line scans of the phase shifts for the London (a) and Clem (b) models $\left(\lambda_{\mathrm{L}}=30 \mathrm{~nm}\right.$ and $\left.\xi_{\mathrm{v}}=40 \mathrm{~nm}\right)$ for the following values of the parameters: angles $\alpha=\pi / 8 ; \pi / 4 ; 3 \pi / 8$ and $\pi / 2$ and thicknesses $0 \lambda_{\mathrm{L}} ; \lambda_{\mathrm{L}}$ and $2 \lambda_{\mathrm{L}}$. The single curves with the largest phase shift correspond to the case $\alpha=\pi / 2$, (for which the thickness is irrelevant) and have been calculated from equations (3) and (5). At lower angles, the curves are clustered around the limiting values $\alpha$ (as also predicted by simple geometrical considerations [6], where, however, the complementary angle $\theta=\pi / 2-\alpha$ was chosen), and within the curve clusters larger phase shifts correspond to increasing thickness.

It can be ascertained that whereas the curves at lower thickness seem to converge monotonically to their limiting value, at larger thicknesses a broad maximum appears due to effect of the fluxon core [7]. This result implies that using suitably modified expressions derived from equations (3) and (5), which converge asymptotically to their limiting values, will lead to approximations which are qualitatively different from the observed trends.

\section{Approximation of the Phase Shift and Out-of-Focus Images}

In this section the problem of approximating the one-dimensional line scans of the phase shift by use of equations (3)-(5) is considered, with particular reference to the case of $\alpha=\pi / 4$, which is the more interesting one from the experimental point of view, as it represents a good compromise between the opposite effects of thickness and magnetic field.

Owing to the qualitative differences remarked at the end of the foregoing section, the following criteria have been chosen: first the maximum phase difference of $\pi$ predicted for the case $\alpha=\pi / 2$ has been replaced with the maximum value $\Delta$ taken from the calculated line scans; second, in order to have only one parameter free for the minimum chi-square best fit, we have taken for the Clem model $\frac{\xi_{\mathrm{v}}}{\lambda_{\mathrm{L}}}=\frac{4}{3}=1.333$. Remember that the bulk value for $\lambda_{\mathrm{L}}$ used in the former numerical calculations has been chosen equal to $30 \mathrm{~nm}$.

With these assumptions, the one-dimensional scans, over a width corresponding to about 20 $\lambda_{\mathrm{L}}$ (which includes the broad maximum as well as the slowly decreasing part of the phase), are approximated by the following set of values. For the London model, at $t=0 \lambda_{\mathrm{L}}, \Delta=0.5 \pi$ and $\lambda_{\mathrm{L}}=0.0385 \mathrm{~nm}$, at $t=\lambda_{\mathrm{L}}, \Delta=0.526 \pi$ and $\lambda_{\mathrm{L}}=0.0252 \mathrm{~nm}$, and $t=2 \lambda_{\mathrm{L}}, \Delta=0.575 \pi$ and $\lambda_{\mathrm{L}}=0.0215 \mathrm{~nm}$.

For the Clem model it results at $t=0 \lambda_{\mathrm{L}}, \Delta=0.5 \pi$ and $\lambda_{\mathrm{L}}=0.0385 \mathrm{~nm}$, at $t=\lambda_{\mathrm{L}}, \Delta=0.520 \pi$ and $\lambda_{\mathrm{L}}=0.0289 \mathrm{~nm}$, and at $t=2 \lambda_{\mathrm{L}}, \Delta=0.559$ and $\lambda_{\mathrm{L}}=0.0253 \mathrm{~nm}$.

It is interesting to note that the best fit gives for $\lambda_{\mathrm{L}}$ values which are above the bulk value when the thickness is below $\lambda_{\mathrm{L}}$, and below the bulk value in the opposite case. Over the range of thicknesses investigated, $\lambda_{\mathrm{L}}$ varies at the maximum of about $\pm 30 \%$. Obviously, the best fit is better for lower thicknesses, but in the worst case, the maximum error in the phase does not exceed $0.006 \pi$.

The values found from the best fit procedure have been inserted as input in programs calculating the out-of-focus image, and compared with the results obtained from the two-dimensional calculations. The results are shown in Figures 4 and 5, which refer to the London and Clem cases, respectively. The left hand side (LHS) of the Figure reports the two-dimensional defocus images, at a defocus distance of $2.5 \mathrm{~mm} \mathrm{(a),} 5 \mathrm{~mm}$ (b), $7.5 \mathrm{~mm} \mathrm{(c)} \mathrm{and} 10 \mathrm{~mm}$ (d); the middle part the line scan across the center of the images and the right hand side (RHS) the line scan resulting from the one-dimensional calculation. It can be seen that the overall agreement is rather good, although some differences could be detected, namely the maxima and minima are slightly lower in the one-dimensional simulation, which on the other hand presents secondary maxima with more contrast. 

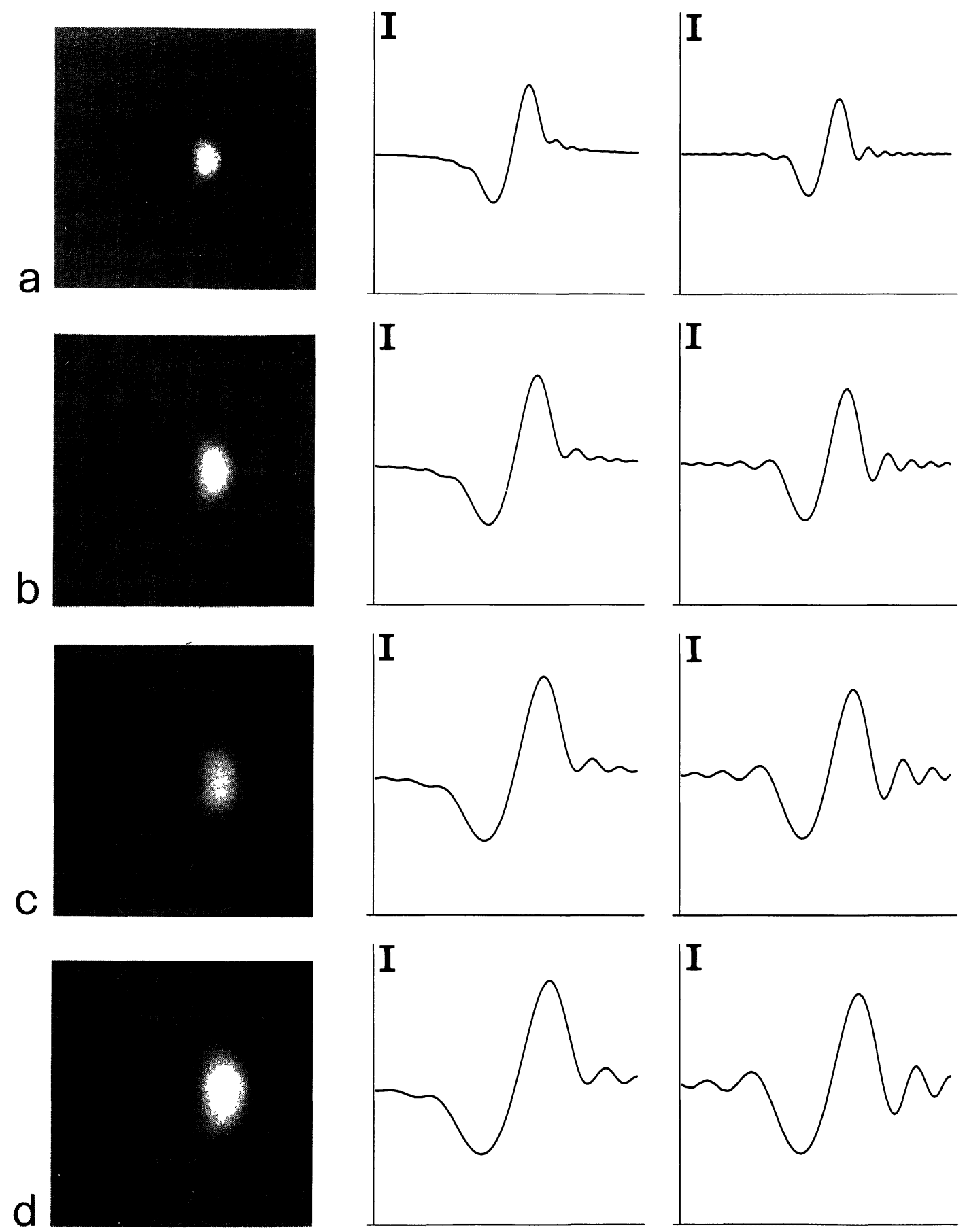

Fig. 4. - London model vortex simulations. Out-of-focus images for a London vortex at $2.5 \mathrm{~mm}(\mathrm{a}), 5 \mathrm{~mm}$ (b), $7.5 \mathrm{~mm}$ (c) and $10 \mathrm{~mm}$ (d) are shown on the left. Their corresponding line scans are in the middle, whereas line scans resulting from the 1-D calculation are shown on the right. Note that the 1-D scans reveal a diminished intensity variation across the fluxon core as well as more pronounced secondary maxima. Calculation parameters: $\alpha=\pi / 4, t=\lambda_{\mathrm{L}}$. Total image width in abscissa $20 \lambda_{\mathrm{L}}$; intensity range in ordinate $0-2$. 

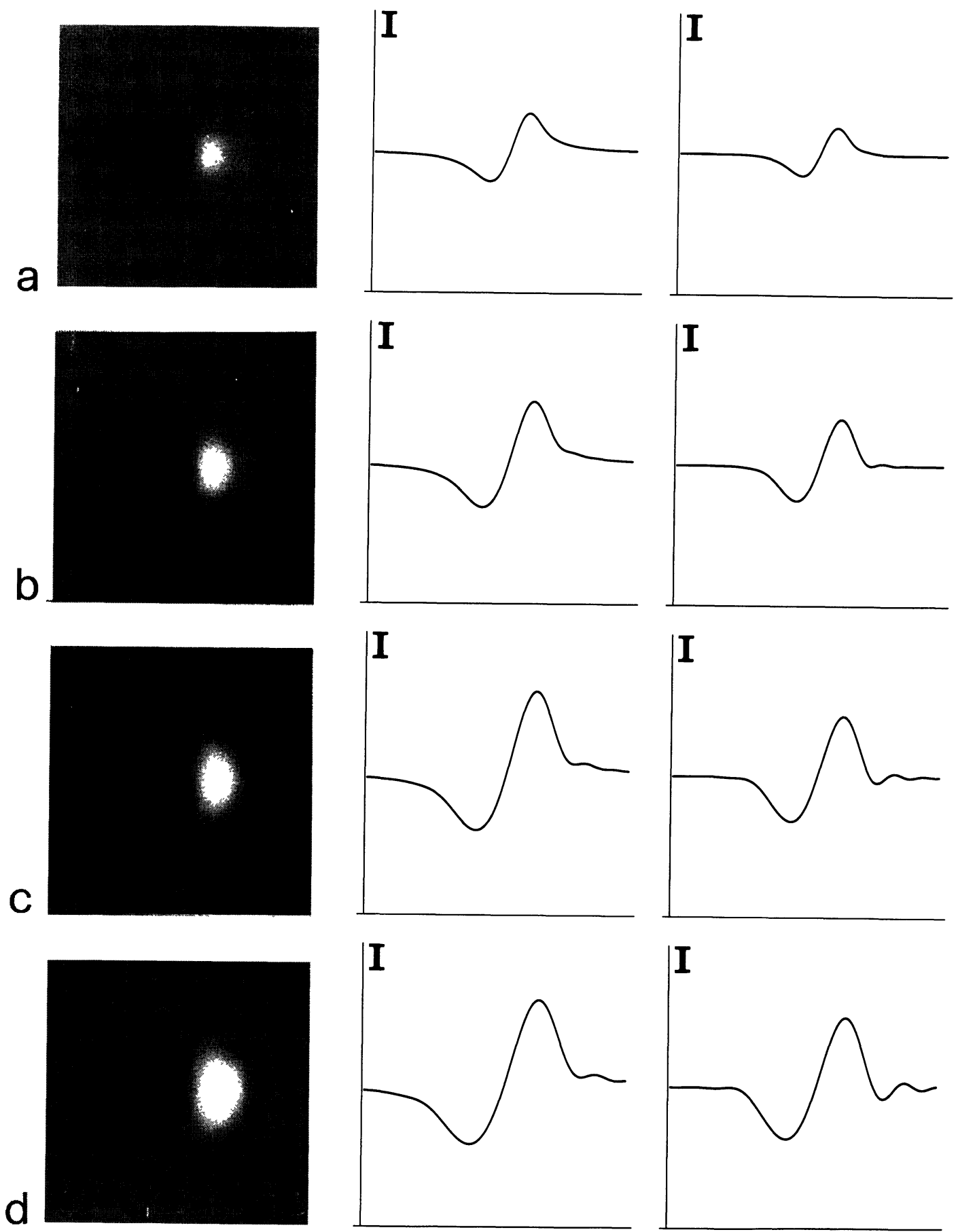

Fig. 5. - Clem model vortex simulations. Out-of-focus images for a Clem vortex at $2.5 \mathrm{~mm}$ (a), $5 \mathrm{~mm}$ (b), $7.5 \mathrm{~mm}$ (c) and $10 \mathrm{~mm}$ (d) are shown on the left. Their corresponding line scans are in the middle, whereas line scans resulting from the 1-D calculation are shown on the right. Again note that the 1-D scans reveal a slightly diminished intensity variation across the fluxon core and more pronounced secondary maxima than the 2-D simulations. Overall, the Clem model presents features with less contrast than the London model. In this case $\xi_{\mathrm{v}}=40 \mathrm{~nm}$. Total image width in abscissa $20 \lambda_{\mathrm{L}}$; intensity range in ordinate $0-2$. 
In view of the fact that we are trying to approximate the central part of a two-dimensional pattern with a one-dimensional model, we may conclude that the agreement is rather satisfactory.

One advantage of having analytical one-dimensional approximations is that it is possible to investigate by means of the stationary phase approximation the transition from the geometric to the optical domain [14]. This can be done by defining a particular defocus at which the geometric optic caustic tip is formed [15]. This defocus distance can be taken as an indication of the power of the phase object. For the London model, with maximum phase difference $\Delta$, this defocus distance can be easily calculated and is given by

$$
Z_{\mathrm{c}}=\frac{4 \pi \lambda_{\mathrm{L}}}{\lambda_{\mathrm{e}} \Delta}
$$

The corresponding value of the dimensionless generalized defocus parameter $p_{\mathrm{c}}=\lambda_{\mathrm{e}} Z_{\mathrm{c}} / \lambda_{\mathrm{L}}^{2}$ turns out to be $4 \pi / \Delta$, which, being $\Delta \simeq 0.5 \pi$, gives $p_{\mathrm{c}} \simeq 8$. Moreover, the quadratic dependence of $Z_{\mathrm{c}}$ on $\lambda_{\mathrm{L}}$ shows that for high $T_{\mathrm{c}}$ superconductors very large defocus distances are necessary, in order to have detectable contrast. It should be noted that the value for the generalized defocus corresponds to region of the curve in Figure 8 of reference [2] where the curves are bending towards the slowly varying region. Although these curves are strongly influenced by diffraction (according to the Wohlleben criterion $[14,16]$ it has little sense to speak about geometric optical realm when the phase difference is about $\pi$ ), the calculated values nonetheless gives the correct order of magnitude and the correct functional dependence on the experimental parameters.

\section{Model Discrimination}

Although the physical assumptions behind the London and Clem models support the latter as the more suitable for interpreting the experimental data, the question may arise whether it is possible to discriminate between the two models on the basis of the experimental data alone.

Let us examine this possibility with respect to the out-of-focus and holographic observations. In the first case, a comparison between Figures 4 and 5 suggests that the main difference between the predictions of the two models lies in the contrast of the secondary rings surrounding the bright and dark globules. In particular the London model gives rise to more contrasty features.

Now the experimental patterns recorded so far show that these secondary rings are often absent or, when present, have very faint contrast. Does this observation conclusively favor the Clem model? Unfortunately the answer is negative, since experimental patterns do not correspond precisely to the mathematical simulations, but are influenced by several other detrimental factors, such as the partial coherence of the illuminating beam and the presence of inelastic scattering effects.

A rather easy and quick assessment of the influence of these two detrimental factors can therefore be gained by using the one-dimensional model, in combination with modern computers and the availability of a high level language like Mathematica [17], which allows the writing of transparent codes and has outstanding graphics capabilities.

First, the intensity is calculated for the perfect coherent, plane wave case using FFT methods; then this intensity is convoluted by the intensity distribution of the effective source, whose angular distribution $I(\alpha)$ can be described, according to Guigay (private communication and [18]), by the sum of two Gaussian functions, simulating the angular source size and the inelastic scattering broadening respectively, i.e.

$$
I(\alpha)=r_{\mathrm{el}} \exp \left(-\frac{\alpha^{2}}{\alpha_{\mathrm{el}}^{2}}\right)+r_{\mathrm{in}} \exp \left(-\frac{\alpha^{2}}{\alpha_{\mathrm{in}}^{2}}\right)
$$



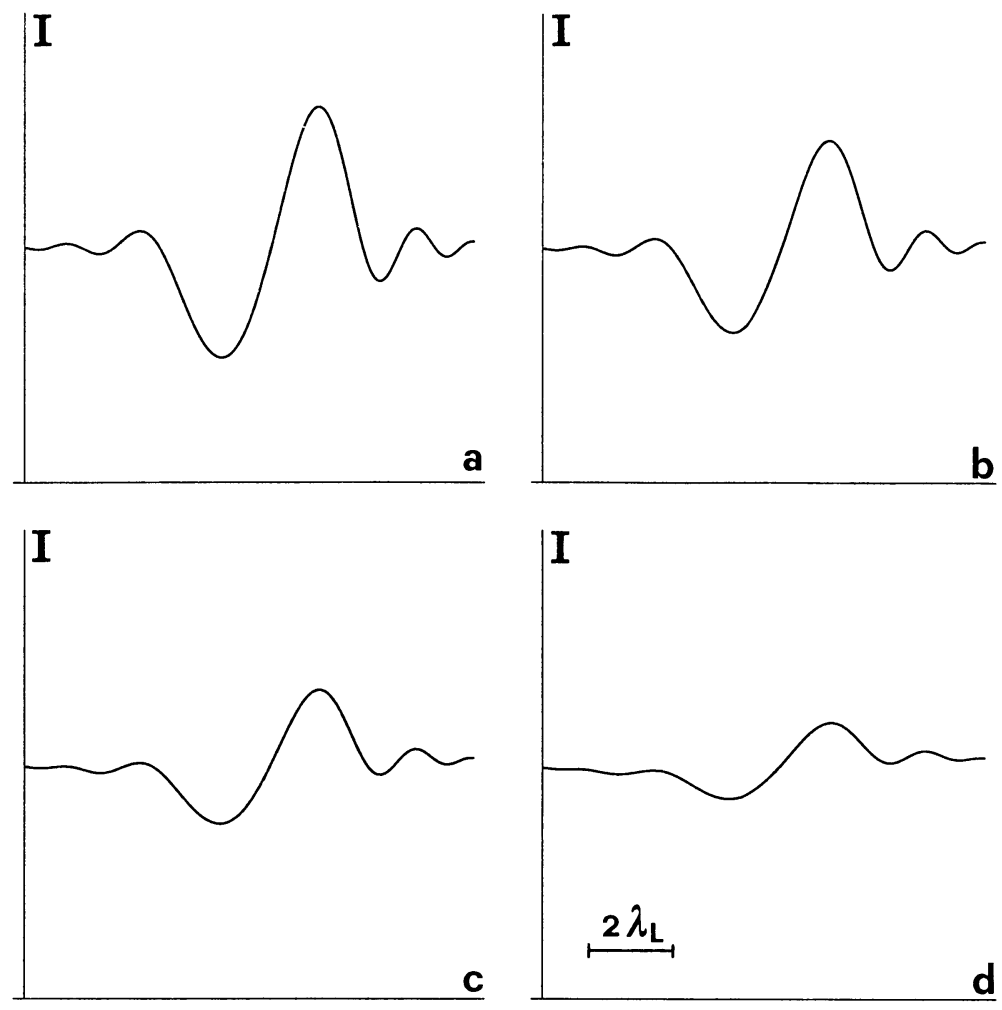

Fig. 6. - Elastic and inelastic scattering effect on image intensity. The 1-D calculation of a London vortex for purely elastic scattering $\left(r_{\mathrm{el}}=1\right)$ in (a), $r_{\mathrm{el}}=0.75(\mathrm{~b}), r_{\mathrm{el}}=0.5$ (c) and $r_{\mathrm{el}}=0.25$ (d). In all cases $\alpha_{\mathrm{el}}=2 \times 10^{-6}$ radians and $\alpha_{\mathrm{in}}=2 \times 10^{-5}$ radians. As expected, inelastic scattering greatly reduces the contrast variation for a London vortex. Total image width in abscissa $20 \lambda_{L}$; intensity range in ordinate $0-2$.

where $r_{\mathrm{el}}+r_{\mathrm{in}}=1$ represents the relative ratios of elastic and inelastic scattering, and $\alpha_{\mathrm{el}}$ and $\alpha_{\mathrm{in}}$ are the corresponding angular widths.

Figure 6 reports the effect of the convolution on the intensity of the London model for the following values of the parameters: $\alpha_{\mathrm{el}}=2 \times 10^{-6} \mathrm{rad}, \alpha_{\mathrm{in}}=2 \times 10^{-5} \mathrm{rad}$ and $r_{\mathrm{el}}=1$ (a) (only partial coherence effects taken into account); $r_{\mathrm{el}}=0.75$ (b); $r_{\mathrm{el}}=0.5$ (c) and $r_{\mathrm{el}}=0.25$ (d). It can be ascertained that, as expected, the contrast of the secondary maxima decreases when the inelastic scattering ratio increases. It should be noted that the simulations require only a few minutes, so that a great variety of theoretical conditions can be tested, with all the parameters varied independently.

The conclusion is that it is extremely difficult to discriminate between the London and Clem model on the basis of their out-of-focus patterns, especially if partial coherence and inelastic scattering effects are taken into account; brighter prospects to achieve such discrimination are however linked to the electron holography analysis.

The superiority of electron holography [5] with respect to the standard phase-contrast methods grouped under the heading of Lorentz microscopy [4] lies in the fact that at the end of the reconstruction process the true object phase difference is available in optical or numerical form. In this latter case, the trend of the phase across the vortex can be extracted with high accuracy, and compared with the theoretical predictions. 

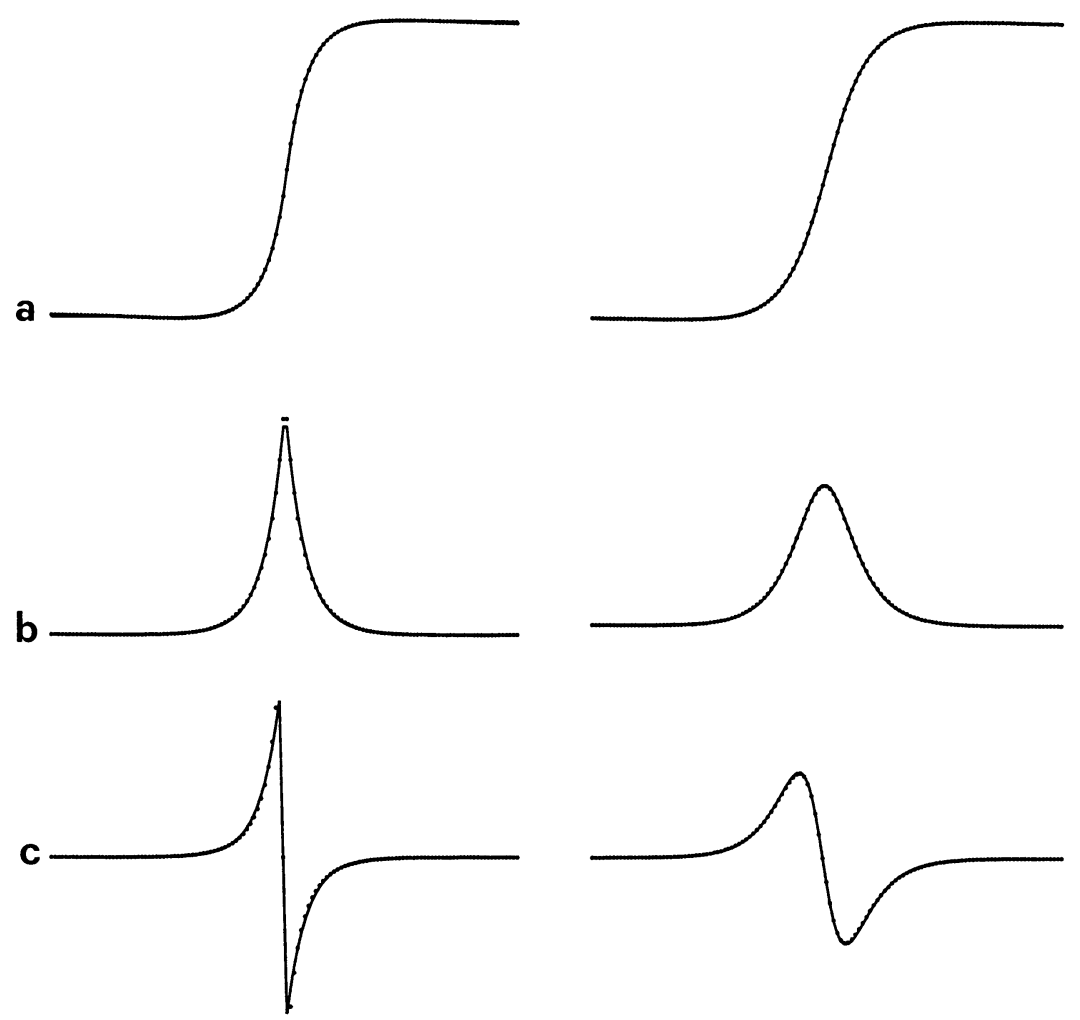

Fig. 7. - Comparison between the values of the phase (a), the first (b) and the second (c) derivatives of the phase shift, calculated from the one-dimensional scans (dotted) and their approximations (continuous line) both for the London (left hand side) and Clem (right hand side) models. The case of $\alpha=\pi / 4$ and $t=\lambda_{\mathrm{L}}$ has been considered. The best fit for the phase data also fits well to the 1 st and 2 nd derivatives. Total image width in abscissa $20 \lambda_{\mathrm{L}}$; arbitrary units in ordinate.

Figure 7 reports the comparison between the values of the phase (a), the first (b) and the second (c) derivatives of the phase shift, calculated from the one-dimensional scans (dotted) and their approximations (continuous line) both for the London (left column) and Clem (right column) models. The case $\alpha=\pi / 4$ and $t=\lambda_{\mathrm{L}}$ has been considered.

The first comment is that the best fit for the phase gives also good approximations for the derivatives. Moreover, it can be ascertained that differences between the curves representing the phase are not detectable as they are smaller than the experimental error. A small difference is detectable for the London model in the derivatives at the center. By comparing the trend of the phases of the London and Clem model, Figure 7a, it should be noted that the difference is larger, but still of the same order of magnitude, of the experimental error, so that their discrimination is difficult. In spite of this similarity, the two models are qualitatively very different, since the London model presents a cusp in the first derivative and an abrupt jump in the second one, whereas the Clem model has a continuous and broader trend, see Figures $7 \mathrm{~b}$ and $7 \mathrm{c}$.

It should be also remarked that the curves in Figure $7 \mathrm{~b}$ are very similar to the trend of the field across the core as predicted by the two models, and this similarity is not entirely casual as the relation between field and phase is given by an integral/derivative.

On this basis it seems that discrimination can be easily performed by looking at the derivative 
of the experimentally reconstructed phase. However it should be remarked that in the optical or digital reconstruction the spatial frequency spectrum is filtered, which affects the hologram resolution.
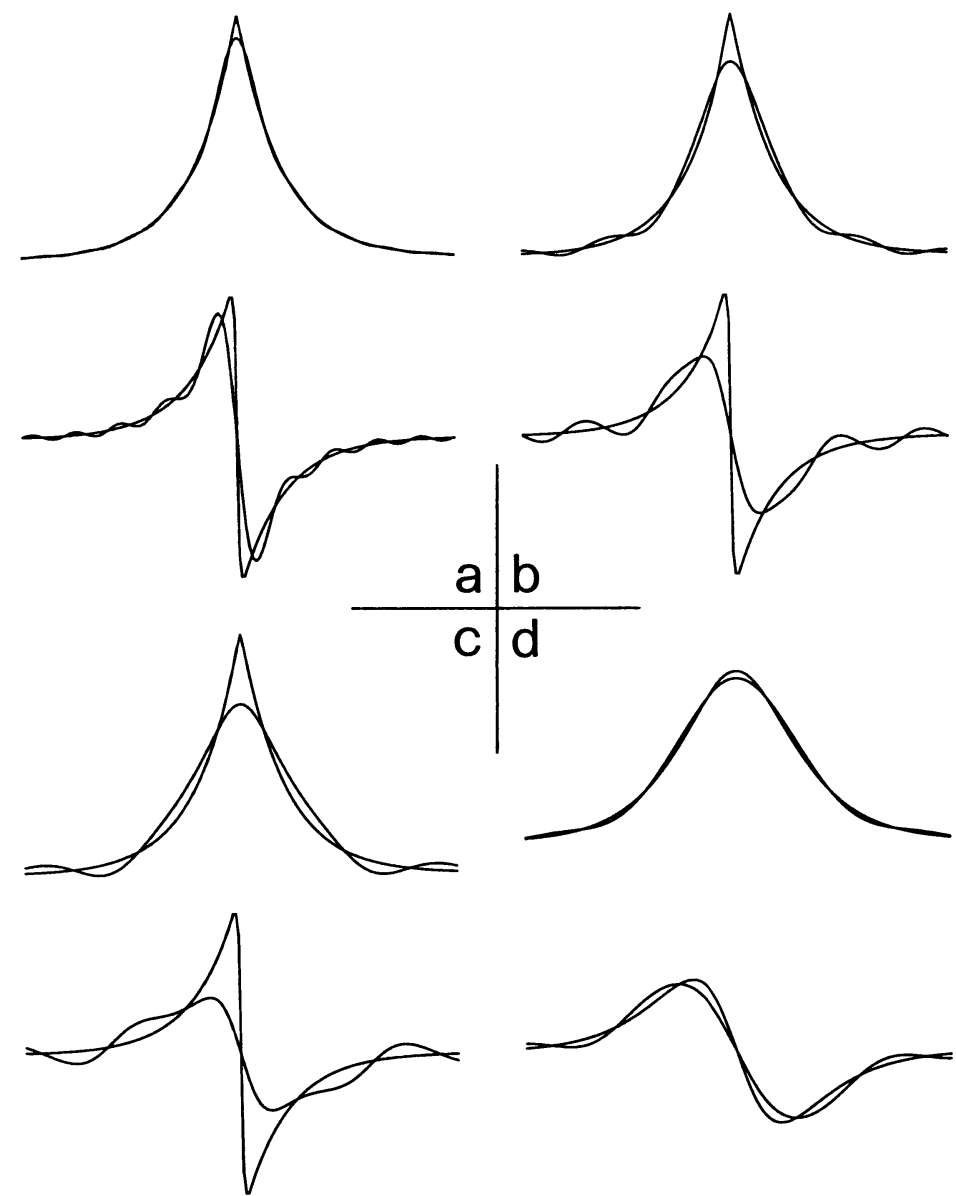

Fig. 8. - Effect of resolution on the reconstructed phase data. The 1-D model of a London vortex convolved with apertures of size $\lambda_{\mathrm{L}}(\mathrm{a}) ; 2 \lambda_{\mathrm{L}}$ (b) and $3 \lambda_{\mathrm{L}}$ (c). The upper curves denote the 1st derivative whereas the lower curves are the 2 nd derivative. The effects of resolution on a Clem vortex are revealed only for the largest aperture $3 \lambda_{\mathrm{L}}(\mathrm{d})$ indicating that model discrimination is possible with high resolution phase data. Line scan width $=0.3 \mu \mathrm{m}=10 \lambda_{\mathrm{L}} ;$ arbitrary units in ordinate.

Also in this case, the effect of the resolution on the reconstructed phase can be easily taken into account for the one-dimensional model, since by a direct Fourier Transform (FT) the spectrum is calculated, then filtered by the reconstruction aperture followed by an inverse FT to obtain the reconstructed wavefunction, from which the amplitude and especially the phase are extracted. Figure 8 shows the result of these operations for the case $\alpha=\pi / 4$ and $t=\lambda_{\mathrm{L}}$, with reconstruction apertures corresponding to a resolution of (a) $\lambda_{\mathrm{L}}$; (b) $2 \lambda_{\mathrm{L}}$ and (c) $3 \lambda_{\mathrm{L}}$. The same calculations, carried out for the Clem model, show no influence of the resolution, except for the largest value of $3 \lambda_{\mathrm{L}}(\mathrm{d})$. It can be concluded that, insofar as the resolution is of the same order as $\lambda_{\mathrm{L}}$, the discrimination between the models seems possible. 


\section{Conclusions}

The results obtained in this paper show that:

i) one-dimensional analytical models for the London and Clem topographies can be profitably used in the fitting of the experimental data, allowing a more accurate estimate of an important parameter like the London wavelength;

ii) the same models can be used for quick and easy one-dimensional simulation of the factors affecting the experimental conditions, as demonstrated by the influence of partial coherence and inelastic scattering on the out-of-focus images and of the resolution on the reconstructed phase.

The results obtained in the one-dimensional simulation can therefore serve to acquire a deeper understanding of the phenomena and as a guide for the more accurate, but time and memory consuming, two-dimensional simulations.

\section{Acknowledgements}

The authors gratefully acknowledge Dr. A. Fukuhara, Dr. U. Kawabe and Dr. T. Onogi of Hitachi and Dr. Q. Ru of the Tonomura Electron Wavefront Project, ERATO-JRDC, for their stimulating discussions and Dr. J. Endo, T. Furutsu, Dr. M. Igarashi, Dr. H. Kajiyama, Dr. S. Kondo, S. Kubota, S. Matsunami, N. Moriya and Dr. N. Osakabe of Hitachi, for their technical assistance. This work has been done within a collaboration scheme of one of the authors (G.P.) with Dr. A. Tonomura and his group at the Hitachi Advanced Research Laboratory, Japan. Extraordinary financial support from the University of Bologna for computing facilities is gratefully acknowledged. This work has been partially supported by funds from MURST coordinated by Consorzio INFM and CNR-GNSM. Useful discussions with Prof. G. F. Missiroli, Dr. G. Matteucci, Department of Physics, and Dr. A. Migliori, LAMEL-CNR, as well as the skillful technical assistance of S. Patuelli are gratefully acknowledged.

\section{References}

[1] Harada K., Matsuda T., Bonevich J.E., Igarashi M., Kondo S., Pozzi G., Kawabe U. and Tonomura A., Nature 360 (1992) 51.

[2] Bonevich J.E., Harada K., Kasai H., Matsuda T., Yoshida T., Pozzi G. and Tonomura A., Phys. Rev. B 49 (1994) 6800.

[3] Harada K., Kasai H., Matsuda T., Yamasaki M., Bonevich J.E. and Tonomura A., Jpn. J. Appl. Phys. 33 (1994) 2534.

[4] Chapman J.N., J. Phys. D 17 (1984) 623.

[5] Tonomura A., Electron Holography (Springer- Verlag, Berlin, 1993).

[6] Bonevich J.E., Harada K., Matsuda T., Kasai H., Yoshida T., Pozzi G. and Tonomura A., Phys. Rev. Lett. 70 (1993) 2952.

[7] Bonevich J.E., Harada K., Kasai H., Matsuda T., Yoshida T. and Tonomura A., Phys. Rev. B 50 (1994) 567.

[8] Capiluppi C., Pozzi G. and Valdrè U., Philos. Mag. 26 (1972) 865.

[9] Migliori A., Pozzi G. and Tonomura A., Ultramicrosc. 49 (1993) 87.

[10] Pozzi G., Bonevich J. E. and Tonomura A., Proc. XIIIth Internat. Congr. on Electron Microsc., Vol 1 (1994) p. 309. 
[11] Pozzi G., Bonevich J. E. and Tonomura A., Proc. XIIIth Internat. Congr. on Electron Microsc., Vol 1 (1994) p. 339.

[12] Huebener R.P., Magnetic Flux Structures in Superconductors (Springer, Berlin, 1979).

[13] Guigay J.P. and Bourret A., C. R. Acad. Sci., Paris 264 (1967) 1389.

[14] Wade R., in Electron Microscopy in Materials Science, U. Valdrè Ed. (Academic Press, New York, 1971) p. 681.

[15] Merli P.G. and Pozzi G., Optik 51 (1978) 39.

[16] Wohlleben D.J., in Electron Microscopy in Materials Science, U. Valdrè Ed. (Academic Press, New York, 1971) p. 713.

[17] WOLFRAM RESEARCH, Mathematica V.2.2, Wolfram Research, Inc., Champaign, Illinois, (1994).

[18] Guigay J.P., Proc. VIth Europ. Congr. on Electron Microsc. (1976) p. 300. 